\title{
Second and Third Order Adaptable Threshold for VAD in VoIP
}

\author{
Abhijeet Sangwan", H.S.Jamadagni”, Chiranth M.C.', Rahul Sah", R. Venkatesha Prasad”, Vishal Gaurav" \\ "Dept. of Electronics \& Communication, PESIT, " Center for Electronic Design Technology, IISc, Bungalore \\ Email: sangwan-a@yahoo.com. chiranth@symonds.net, rahulsah79@mail.com., vishalgaurav78@yahoo.co.uk, \\ (hsjam, vprasad\}@ cedt.isc.ernet. in
}

\begin{abstract}
Reducing the Internet traffic is a requirement to share the bandwidth wirh many sessions. When circuit switched applications are ported on to the Internet, bandwidth saving becomes all the more prominent. An efficient adaptable threshold VAD (Voice Activity Detection) for Voice over IP systems are presented in rhispaper. The discussion includes two energy-based algorithm with higher order threshold refreshing schemes. The performance of all the schemes nrepresented, the results bring out clearly the usefulness of the schemesfor VoIP applications with saving in bandwidth.
\end{abstract}

\section{Introduction}

Services offered by Public Switched Telephone Networks (FSTN) systems are expensive when the distance between the calling and called subscriber is large. The current trend is to provide this service on data networks. Data networks work on the best effort delivery and resource sharing through statistical multiplexing. Hence the cost of services compared to circuitswitched networks is considerably less. However, these networks do not guarantee faithful voice transmission. Voice over IP (VolP) systems have to ensure that voice quality does not significantly deteriorate due to network conditions such as packet-loss and delays. Providing Toll Grade Voice Quality [4] through VolP systems remains a challenge.

In this paper we concentrate on the problem of reducing the required bandwidth for $\boldsymbol{a}$ telephone-like voice connection on Internet using Voice Activity Detection $\mathrm{VAD}$ ), while maintaining the voice quality. VAD is used in Voice Recognition systems, Compression and Speech coding $[3,11,5]$ which are non real-time applications.

It VoIP systems the voice data (or payload for packet) is transmitted along with a header on a network. The header size in case of Real Time Protocol (RTP, [8]) is 12 bytes. The ratio of header to payload size is an important factor for selecting the payload size for a better throughput from the network. Lower size payload helps in a better real-time quality, but decreases the throughput. Alternately, higher size payload gives more throughput but performs poorly in real-time. A constant payload size representing a segment of speech is referred to as a 'Frame' in this paper. The frame size is determined by the above considerations. If a frame does not contain a voice signal it need not be transmitted. The VAD for VolP has to determine if $a$ frame contains a voiced signal. The decision by VAD algorithms for VolP is always on a frame-by-frame basis.
In this paper, time-domain energy-based algorithms are presented with varied complexity and quality of speech. Mainly, time domain techniques are discussed with the intention of lowering the computational complexity involved. Results obtained, and an exfaustive comparison of various algorithms with quantitative measurements of speech quality is presented. We focus on improving the performance by using higher order systems in place of the first-order systems used in [9]. There are many previous studies on VAD that dealt with energy-based algorithms such as [7]. In this paper, a procedure for choosing the scaling parameter ' $\mathrm{p}$ ' [7] is also' given for higher order system. We restrict our study to time domain algorithms as it is faster for implementation in real time systems compared to frequency domain solutions [1]

\subsection{Characteristics of Speech}

Conversational speech is a sequence of contiguous segments of silence and speech [2]. VAD algorithms employ some form of speech pattern classification to differentiate between voice and silence periods. Identifying and rejecting transmission of silence periods helps reduce Internet traffics.

\subsection{Desirable aspects of VAD algorithms:}

- A Good Decision Rule: A physical property of speech that can be exploited to give consistent judgement in classifying segments of the signal into silence or otherwise.

- Adaptability to changing background noise: Adapting to non-stationary background noise improves robustness, especially in wireless telephony.

- Low computational complexity: Internet telephony is a realtime application. Therefore the complexity of VAD algorithm must be low to suit real-time applications.

\section{Parametersfor VAD Design}

The differentiation of the voiced signal into speech and silence is done on the basis of speech characteristics. The signal is sliced into contiguous frames. A real-valued non-negative parameter, the average energy content, is associated with each frame. If this parameter exceeds a certain threshold, the signal frame is classified as ACTIVE; else it is INACTIVE. We refer to these INACTIVE frames also as noise frames.

\subsection{Choice of Frame Duration}

VoIP receivers may queue up incoming packets in a packeibuffer that allows them to play audio even if incoming packets are delayed due to network conditions.

Consider a VoIP system having a buffer of $3-4$ packets. Having frame duration of $10 \mathrm{~ms}$ allows the VolP system to start playing the audio at the receiver's end after 30 to $40 \mathrm{~ms}$ from the time the 
queue started building up. If the frame duration is $50 \mathrm{~ms}$, there would be an initial delay of $150-200 \mathrm{~ms}$, which is unacceptable. Therefore. the frame duration must be chosen properly. Current VolP systems use $20-40 \mathrm{~ms}$ frame sizes.

The specifications for,encoding speech for all VAJJ algorithms are that of Toll Grade Quality \{4\}:

- $\quad 8 \mathrm{kHz}$ sampling frequency

- 256 levels of linear quantization (8 Bit PCM) [10]

- Single channel (mono) recording.

Advantage of using linear PCM is that the voice data can be transformed to any other compressed code (G711, G723, G729).

Frame duration of $10 \mathrm{~ms}$, corresponding to 80 samples is used.

\subsection{Energy of a Frame}

Let $X(i)$ be the $i^{\text {th }}$ sample of speech. If the length of the frame were $\mathrm{k}$ samples, then the $\boldsymbol{j}^{\text {th }}$ frame can be represented in time domain by a sequence as

$$
f_{j}=\{x(i)\}_{i=(j-1) k+1}^{j k}
$$

We associate energy $E_{\xi}$ with the $j^{\text {th }}$ frame as

$$
E_{j}=\frac{1}{k} \sum_{i=(j-1) k+1}^{j k} x^{2}(i)
$$

where,

$E_{i}$ is the energy of the $i^{\text {th }}$ frame and

$f_{i}$ is the $\mathrm{j}$ " frame that is under consideration

\subsection{Initial Value of Threshold}

A sample that contains only background noise is used to compute the initial threshold value parameter. For example, the initial estimate of energy is obtained by taking the mean of the energies of each frame $\boldsymbol{s}$ in

$$
E_{r}=\frac{1}{v} \sum_{m=0}^{v} E_{m}
$$

where,

$E_{r}=$ initial threshold estimate,

$v=$ number of frames in prerecorded sample.

We have taken a prerecorded sample of 5 secs, i.e., 500 frames. Alternately, it may be assumed that the first 20 frames of the given speech sample are INACTTVE and compute $E_{r}$ from (3) taking $v=20$

\section{VAD Algorithms}

Energy of a frame is a reasonable parameter on the basis of which frames may be classified as ACTIVE or INACTIVE. The energy of ACTIVE frames is higher than that of INACTIVE frames [2]. The classification rule is,

$$
\text { IF } \quad E_{j}>k E_{r} \quad \text { where } k>1
$$

Frame is ACI'IVE

ELSE

Frame is INACTIVE

In this equation, $E_{\text {q }}$ represents the energy of noise frames, while $k E_{r}$ is the 'Threshold' being used in the decision-making. Having a scaling factor, $\mathrm{k}$ allows a safe band for the adaptation of $E_{r}$ and hence, the threshold. We use $k=4$ in all our studies as this gives a fair amount of margin for adaptability.

ACTIVE frames are transmitted; INACTIVE frames are not.

The following algorithms use $\mathrm{Eq}(4)$ as the decision rule. The first order system $i$ is discussed in detail in [9], we discuss it briefly here in connection with the second and third order systems for the sake of completeness.

\subsection{SED Simple Energy-Based Detector}

It is now sufficient to specify the reference noise energy, $\boldsymbol{E}$, for use in $\mathrm{Eq}(4)$ to formulate the schemes completely. Since background disturbance is non-stationary an adaptive threshold is more appropriate. The rule to update the threshold value can be found in [7] as,

$$
E_{\text {mew }}=(1-p) E_{\text {rold }}+p E_{\text {silence }}
$$

Here,

$E_{\text {rnew }}$ is the updated value of the threshold,

$E_{\text {rold }}$ is the previous energy threshold, and

$E_{\text {silence }}$ is the energy of the most recent noise frame.

The reference $E_{\mathrm{r}}$ is updated as a convex combination of the old threshold and the current noise update. $p$ (such that $0<p<1$ ) is chosen considering the impulse response of $\mathrm{Eq} .(5)$ as a furst order filter to he 0.2 [1].

Remarks

- This algorithm is simple to implement. It gave an acceptable quality of speech after compression.

- This algorithm did not give a good speech quality under varying background noise. This was because the threshold of $\mathrm{Eq}$. (5) is incapable of keeping pace with rapidly changing background noise leading to undesirable speech clipping, especially at the beginning and end of speech bursts.

\subsection{AED: Adaptive Energy-Based Detector}

The sluggishness of SED is a consequence of $\mathbf{p}$ in Eq. (5) being insensitive to the noise statistics. We compute $E_{r}$ bassed on second order statistics of INACTIVE frames. A buffer (linear queue) of the most recent ' $m$ ' noise frames is maintained. The buffer contains the value of $\mathbf{E}_{\text {silence }}$ rather than the voice packet itself. Therefore the buffer is an array of $m$ double values. Whenever a new noise frame is detected, it is added to the queue and the oldest one is removed. The variance of the buffer, in terms of energy is given as

$$
\sigma=\operatorname{var}\left[E_{\text {silence }}\right]
$$

A change in the background noise is reckoned by comparing the energy of the new INACI'IVE frame with a statistical measure of the energies of the past ' $m$ ' INACTIVE frames. The variance. just before the addition of a new LNAMVE frame is denoted by $\sigma_{0, \mathrm{k} d}$. After the addition of the new INACTIVE frame the variance is $\sigma_{\text {ner }}$. A sudden change in the background noise implies

\begin{tabular}{|c|c|}
$\sigma_{\text {new }}>\sigma_{\text {oid }}$ \\
\hline $1.25 \geq \frac{\sigma_{\text {new }}}{\sigma_{\text {new }}} \geq 1.25$ & 0.25 \\
\hline $1.10 \geq \frac{\sigma_{\text {new }}}{\sigma_{\text {old }}} \geq 1.00$ & 0.20 \\
\hline $1.00 \geq \frac{\sigma_{\text {new }}}{\sigma_{\text {old }}}$ & 0.15 \\
\hline
\end{tabular}

Table 1: Value of p dependent on $\underline{\sigma}_{\text {old }}$

$\sigma_{\text {new }}$ 
We set a new rule to vary $\mathbf{p}$ in $\mathrm{Eq}$ (5) in steps as per Table 1. As the value of $p$ is varied the adaptation was more profound. The convex combination (Eq.5) now has its coefficients dependent on variance of energies of INACTIVE frames. We are able to make the otherwise sluggish $\mathrm{E}_{\mathrm{r}}$ respond faster to sudden changes in the background noise. The classification rule for the signal frames continues to be Eq(4). Hence, detection of ACTIVE frames is still energy-based. The obvious limitations are (a) Inability to detect non-plosive phonemes and (b) Low SNR conditions caused undue clippings in the compressed signal, as in SED Algorithm.

\subsection{System Response}

The Z-Tratisforth of $\mathrm{Eq}(5)$ is,

$$
\mathrm{E}_{r}(\mathrm{Z})=(1-\mathrm{p}) \mathrm{Z}^{-\mathrm{i}} \mathrm{E}_{\mathrm{r}}(\mathrm{Z})+\mathrm{p} \mathrm{E}_{\text {noise }}(\mathrm{Z})
$$

The Transfer Function may be determined as,

$$
\mathrm{H}(\mathrm{Z})=\frac{\mathrm{E}_{\mathrm{r}}(\mathrm{Z})}{\mathrm{E}_{\text {noise }}(\mathrm{Z})}=\frac{\mathrm{p}}{1-(1-\mathrm{p}) \mathrm{Z}^{1}}
$$

which represents a first order system. The impulse response of this first order system is given below

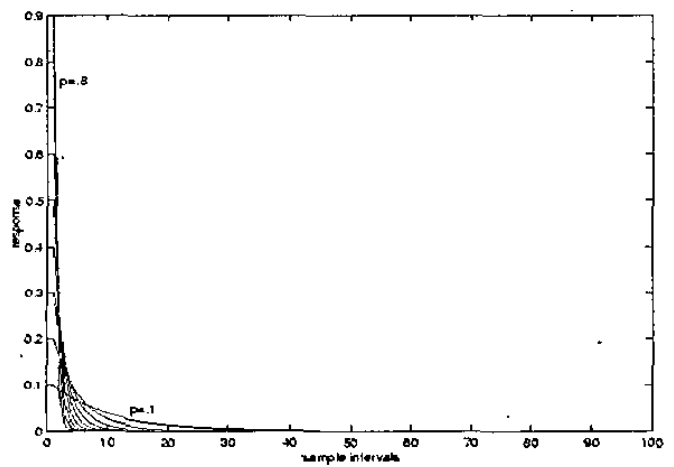

Fig. 1 : Impulse response of Eq (9)

In order to improve the performance, we take higher-order equation, as in Eq (10) second-order and $\mathrm{Eq}$ (11) thud order systems for updating $E_{r}$

$$
\begin{aligned}
& E_{\text {rnew }}=\frac{\left(1-p^{2}\right) E_{\text {rold }}+(1-p) E_{\text {silence-1 }}+p E_{\text {silence }}}{\left(2-p^{2}\right)}(10) \\
& E_{\text {rew }}=\frac{\left(1-p^{2}\right) E_{\text {rold }}+\left(1-p^{2}\right) E_{\text {silence-2 } 2}+(1-p) E_{\text {silence-1 }}+p E_{\text {silence }}}{\left(3-p^{3}-p^{2}\right)}
\end{aligned}
$$

where, $E_{\text {silence, }} E_{\text {silence-1 }}$ and $E_{\text {sivence-2 }}$ are energies of the last three INACTIVE frames, with $E_{\text {rnew }}$ and $E_{\text {rold }}$ having the same meaning as before. In Eq. (5).

$$
\begin{gathered}
H(Z)=\frac{E_{r}(Z)}{E_{\text {silencec }}(Z)}=\frac{p+(1-p) Z^{-1}}{Z-\left(1-p^{2}\right) Z^{-1}} \\
H(Z)=\frac{E_{r}(Z)}{E_{\text {silence }}(Z)}=\frac{\left(1-p^{2}\right) Z^{-2}+(1-p) Z^{-1}+p}{Z-\left(1-p^{3}\right) Z^{1}}
\end{gathered}
$$

The impulse response of the second and third order equation is shown in Fig. 2 and Fig. 3.

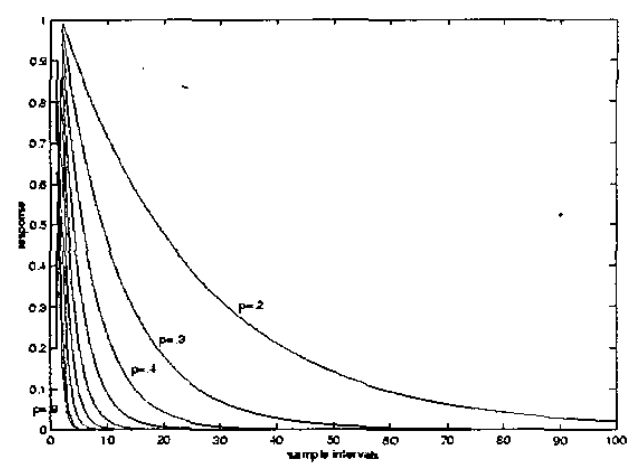

Fig. 2: Impulse response of Eq. (10)

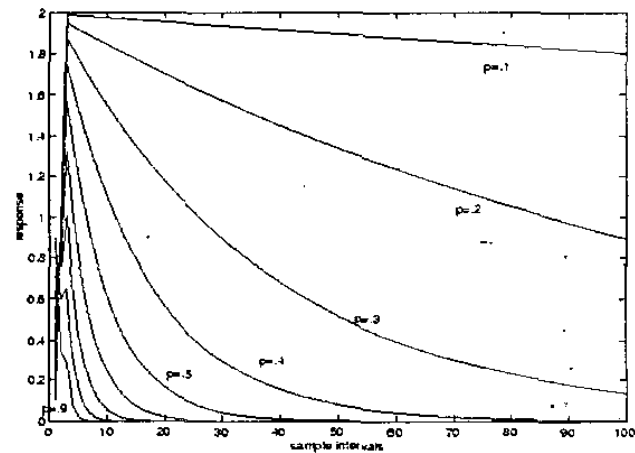

Fig 3. Impulse response of Eq. (11)

As we can seefrom the graphs, the first order system response is very fast, causing the threshold to vary beyond desired limits, whereas the second order system response is optimum, making it a good choice. The third order system response is sluggish in nature, making the threshold less adaptive. Incorporating these changes, we have'four new algorithms for the SED and AED.

\section{Results and Discussions}

The algorithms were tested on various samples. The test templates varied in Loudness, speech continuity, background noise and accent. Both male and female voices have been used. The performance of the algorithms was studied on the basis of the following parameters:

1. Floating Point Operations (FLOPS) required: This parameter is useful in comparing algorithms of their applicability for real-time implementation.

2. Percentage comoression: The ratio of total INACTIVE frames detected to the total number of frames formed expressed as a percentage. A good VAD should have high percentage compression.

- Subiective Speech Ouality: The quality of the samples was rated on a scale of 1 (poorest) to 5 (best) where 4 represents toll grade quality. The input signal was taken to have speech quality 5 . The speech samples after compression 

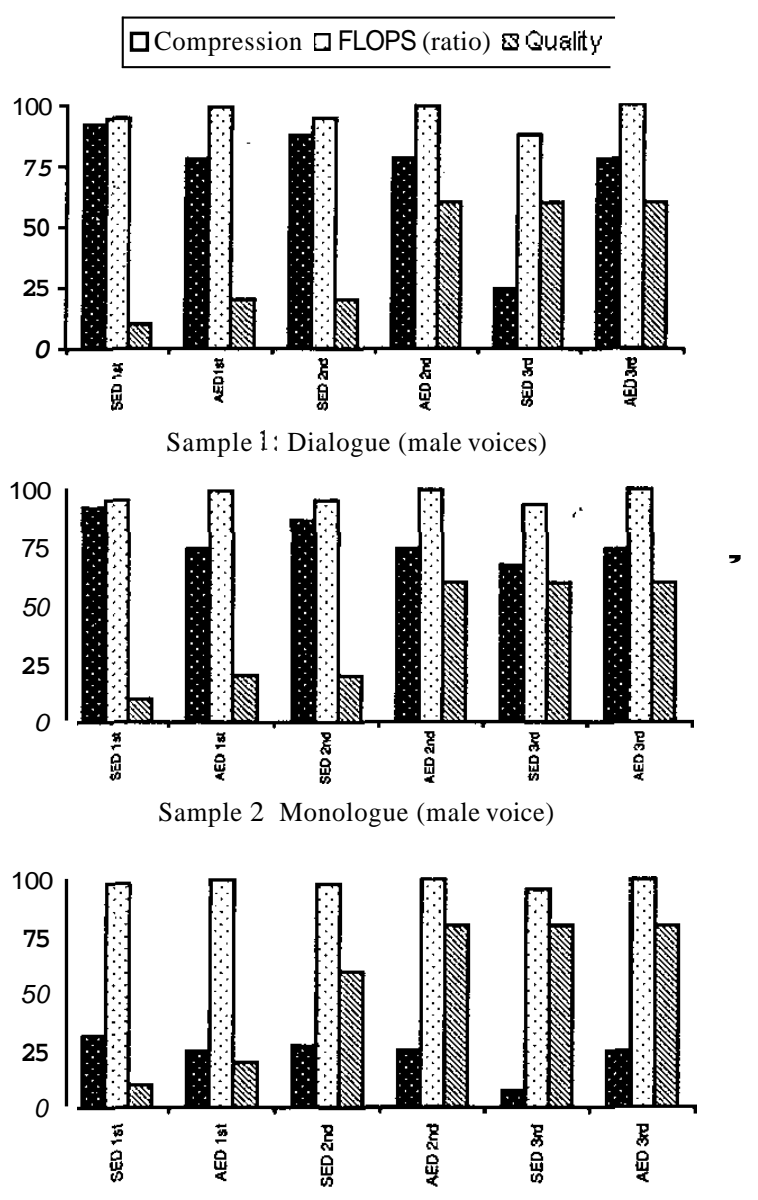

Sample 3: Monologue (female voice)

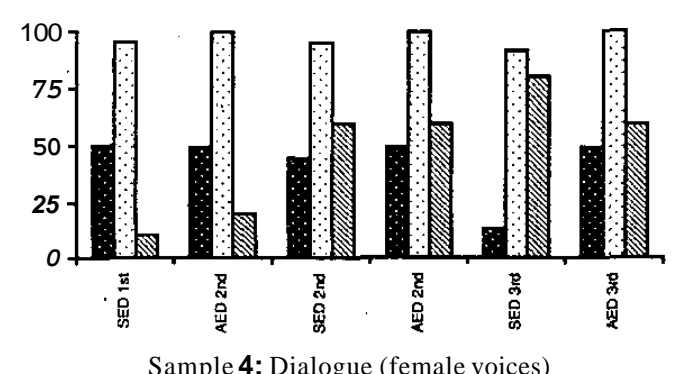

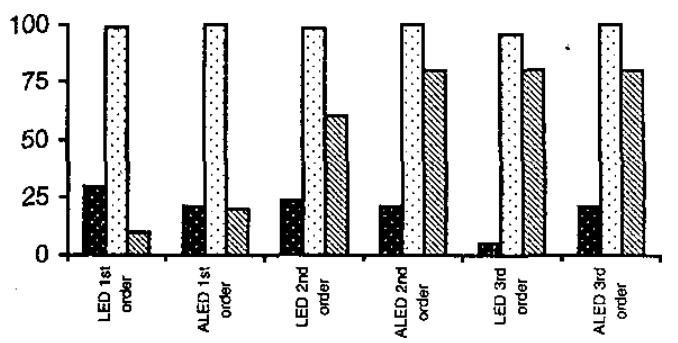

Sample 5: Accented monologue (female voice)

\section{Conclusions}

VolP has become a reality, yet not in common use. This is predominantly due to existing systems being 'not very satisfactory or dependable. A practical solution lies in efficient VAD schemes. The time domain VAD algorithms are found to be computationally less complex. With these schemes, good speech detection and noise immunity were observed. There is still a degradation of performance under low SNR conditions. Compared to first order threshold-refreshing system, second and third order performs better. The algorithms presented in this paper are found to be suitable for real-time applications, with a reasonable quality of speech. For better quality of speech, frequency domain solutions are necessary but it comes with added computational complexity.

\section{References}

[1] A. Sangwan, Chiranth M. C. R. Shah, V. Gaurav, R. Venkatesha Prasad 'Voice Activity Detection for VoP. Time and Frequency domain Solutions". Tenth annual LEEE Symposium on Multimedia Communications and Signal Processing, Bangalore, Nov 2001, pp 20.24.

[2] - B. Gold and N. Morgan, Speech and Audio Signal Processing, John Wiley Publications.

[3] Jongseo Sohn. Nam Soo Kim and Wonyong Sung, "A statistical model-based voice activity detection". IEEE signal processing letters, yol 6, no. 1, January 1999

[4] Kamilo Feher, Wireless Digitol Communications, Prentice Hall India, 2001

[5] Khaled El-Maleh and Peter Kabal, "Comparison of voice activity detection algorithms for wireless personal communications systems". EEEE Canadian Conferenceon Electrical and Computer engineering". May 1997. pp 470-473

[6] L.R Rabiner and M.R. Sambur, An Algorithm for detennining endpoints of isolated utterances, Bell Technical Journal. Feb 1975,pp 297-315.

[7] Petr Pollak and Pavel Sovka, and Jan Uhlir," Noise Suppression System for a Car". proc, of the third European Conference on Speech, Communication and Technology -EUROSPEECH 93, Berlin, Sept 1993. pp 1073-1076

[8] RTP, Real Time Protocol. RFC 1889. www.ietf.org/rfc/rfc1889.uxt

[9] Venkatesha Prasad. et, al. "Comparison of Voice Activity Detection Algorithms for VoIP", accepted fur publication at the Seventh IEEE Symposium on Computers and Communication, July 2002, Taomina, Italy.

[10] Xie and Reddy - Enhancing VoIP designs with PCM Coders. Communication System Design Magazine. San Francisco, California.

[11] Y.D.Cho, K.Al-Naimi and A.Kondoz, "Mixed Decision-Based Noise Adaption for Speech Enhancement". LEEE Electronics Letters Online No. 20010368, 6 Feb 2001 